\title{
ARTí́CULO
}

\section{Efecto de la temperatura sobre el desarrollo embrionario y larval de Graus nigra (Kyphosidae) del norte de Chile}

\author{
Temperature effect on the early development of Graus nigra
}

(Kyphosidae) off northern Chile

\section{Cristian Azocar ${ }^{1}$, Gabriel Claramunt ${ }^{1,2}$, Fernando Yañez $^{1 \dagger}$ y Masatoshi Futagawa $^{1}$}

\author{
${ }^{1}$ Facultad de Recursos Naturales Renovables, Universidad Arturo Prat, Casilla 121, Iquique Chile. cazocars@gmail.com \\ ${ }^{2}$ Instituto de Ciencias Exactas y Naturales (ICEN), Universidad Arturo Prat, Iquique, Chile \\ †septiembre de 1977-septiembre de 2007
}

\begin{abstract}
The rocky fish Graus nigra (Kyphosidae) commonly named mulata, is an important resource for artisanal and recreational fishery off northern Chile, but unfortunately shows show signs of depletion in terms of abundance and size, so have been developing initiatives to its aquaculture with repopulation potential actions. In this paper we report a study of the thermal tolerances in the early stages of development. Fertilized eggs of Graus nigra (Kyphosidae) were obtained from captive adults and incubated at different temperatures between 12 and $22^{\circ} \mathrm{C}$ to study the effect on the development rate, hatching success and survival of early-stages until the total absorption of the yolk. The highest percentage of hatched eggs occurred between 14 and $18^{\circ} \mathrm{C}$. The highest survival was observed between 16 and $18^{\circ} \mathrm{C}$. Yolk exhaustion was observed after $128.3 \mathrm{~h}$ at $12^{\circ} \mathrm{C}, 48.6 \mathrm{~h}$ at $18^{\circ} \mathrm{C}$ and $51.2 \mathrm{~h}$ at $20^{\circ} \mathrm{C}$. A significant reduction in the larvae sizes was observed after 28 to 30 hours post-hatching at $18^{\circ} \mathrm{C}(4.32$ to $2.75 \mathrm{~mm})$ and $20^{\circ} \mathrm{C}(4.18$ to $2.99 \mathrm{~mm})$. The incubation experiments suggest that the optimal temperature for maximizing the eggs hatching success, growth and survival of early larvae is $16^{\circ} \mathrm{C}$, which is coincident with the temperature of maximum spawning intensity in the adult broodstock.
\end{abstract}

Key words: Graus nigra, temperature, development, eggs, larvae

Resumen.- El pez de roca Graus nigra (Kyphosidae), conocido como mulata, es un importante recurso para la pesca artesanal y deportiva de la zona norte de Chile, pero lamentablemente muestra signos de caída en su abundancia y en los tamaños de los peces capturados, por lo que se han desarrollado iniciativas tendientes a su cultivo con fines comerciales y de posibles acciones de repoblamiento. La temperatura es una de las variables ambientales más influyente en el desarrollo embrionario, éxito en la eclosión de huevos y supervivencia larval de peces. Huevos fertilizados de Graus nigra (Kyphosidae) fueron obtenidos de reproductores en cautiverio e incubados a distintas temperaturas entre 12 y $22^{\circ} \mathrm{C}$, para estudiar el efecto sobre la tasa de desarrollo, éxito en la eclosión y supervivencia de estadios tempranos hasta el término del vitelo. El mayor porcentaje de huevos eclosionados ocurrió entre 14 y $18^{\circ} \mathrm{C}$. La mayor supervivencia fue observada entre 16 y $18^{\circ} \mathrm{C}$. El agotamiento del vitelo ocurrió a las $128,3 \mathrm{~h}$ a $12^{\circ} \mathrm{C}, 48,6 \mathrm{~h}$ a $18^{\circ} \mathrm{C}$ y $51,2 \mathrm{~h}$ a $20^{\circ} \mathrm{C}$. Se detectó una reducción significativa en el tamaño de las larvas después de $28-30 \mathrm{~h}$ post-eclosión a $18^{\circ} \mathrm{C}(4,32$ a $2,75 \mathrm{~mm})$ y $20^{\circ} \mathrm{C}(4,18$ a $2,99 \mathrm{~mm})$. Los experimentos de incubación, sugieren que la temperatura óptima para la maximización del éxito de eclosión de huevos de esta especie, así como el crecimiento y supervivencia de larvas tempranas es de $16^{\circ} \mathrm{C}$, coincidente con la temperatura de máxima intensidad de desove registrada para los reproductores adultos en cautiverio.

Palabras clave: Graus nigra, temperatura, desarrollo, huevos, larvas

\section{INTRODUCCIÓN}

La temperatura es una de las variables ambientales más influyente en el desarrollo embrionario, éxito en la eclosión de huevos y supervivencia larval de peces (Camus \& Koustikopoulos 1984), lo cual finalmente se puede reflejar en la fuerza del reclutamiento (Jensen et al. 1989, Beachman \& Murray 1990, Blaxter 1992, Galloway et al. 1998, Ojanguren et al. 1999). Los límites de tolerancia térmica determinan no sólo los efectos en la duración de los estadios ontogénicos, sino también el tamaño de las larvas al momento de la eclosión, eficiencia en la utilización del saco vitelino, el inicio de la primera alimentación y posibles deformaciones embrionarias (Bolla \& Holmefjord 1988, Polo et al. 1991, Bermudez \& Ritar 1999, Kamler 2002, Klimogianni et al. 2004). Dentro de los rangos de 
viabilidad, la temperatura tiene 2 consecuencias muy importantes para la supervivencia: Primero, altas temperaturas producen estadios tempranos más cortos debido a tasas de crecimiento rápidas, con la consiguiente reducción de la probabilidad de muerte por depredación, y segundo, la temperatura puede afectar la posibilidad de un ajuste o desajuste de las larvas en la primera etapa de alimentación con el ciclo de producción de alimento en el ambiente (Cushing 1973, Kamler 1992). Antes de la primera alimentación, el vitelo se utiliza para el desarrollo del embrión con una característica eficiencia para cada especie (Bagarinao 1986, Arul 1991, Parra \& Yúfera 2001). Entre los factores que afectan la tasa de absorción del vitelo y su eficiencia en la conversión en tejidos embrionarios, se encuentra su cantidad inicial en la fertilización (dependiendo de las condiciones de la madre) y la temperatura (Howell 1980, Polo et al. 1991, Buckley et al. 2000, Hardy \& Litvak 2004). El agotamiento del vitelo se asocia al inicio de una etapa de mortalidades masivas, ya que está relacionado al inicio de un período crítico (Hjort 1914), en el cual la larva debe encontrar alimento, ya que de no hacerlo entraría en el punto de no retorno (PNR) (Blaxter \& Hempel 1963).

Es así, que el estudio de las tolerancias térmicas de las especies de peces litorales, ayudaría a la comprensión de la distribución, dinámicas y mecanismos de adaptación de huevos y larvas a las variables medioambientales, especialmente en la zona norte de Chile que está expuesta a una alta variabilidad ambiental producto de eventos oceanográficos cálidos (El Niño) y fríos (La Niña). En condiciones consideradas normales (ENSO neutro) se encuentran típicamente temperaturas superficiales del mar de $16^{\circ} \mathrm{C}$ en invierno y $18^{\circ} \mathrm{C}$ en verano.

Graus nigra (Philippi, 1887), pez de roca conocido como 'mulata', es un importante recurso para la pesca artesanal y deportiva de la zona norte de Chile, siendo muy apetecido por su carne. Graus nigra se distribuye latitudinalmente desde el sur del Perú hasta Valdivia en Chile (Vargas \& Pequeño 2004). Sus huevos y larvas son pelágicas, para luego en los estados juveniles asentarse en pozas intermareales durante invierno y primavera (Berrios \& Vargas 2000), siendo estos juveniles, posibles reclutas del período reproductivo del año anterior, para finalmente habitar en el submareal rocoso durante la etapa adulta (Stepien 1990, Varas \& Ojeda 1990, Muñoz \& Ojeda 1997, 1998). Su estrategia reproductiva corresponde a la de un desovador parcial de tipo asincrónico, con un período reproductivo que comprende desde agosto a diciembre (Flores \& Smith 2010). Lamentablemente, esta especie muestra signos de caída en su abundancia y tamaños de extracción, indicando un agotamiento del recurso (Godoy et al. 2010), por lo que se han desarrollado iniciativas para el cultivo de esta especie con fines comerciales y posible repoblamiento (Flores \& Rendic 2011, Muñoz et al. 2012), para lo cual se requieren estudios de sus tolerancias térmicas en las distintas fases de su ciclo vital. El objetivo de este trabajo fue determinar la influencia de la temperatura sobre la tasa de desarrollo embrionario, éxito en la eclosión, crecimiento, absorción de vitelo y supervivencia larval.

\section{MATeRIALES Y MÉtodos}

\section{OBTENCIÓN DE LOS HUEVOS}

Los huevos se obtuvieron de ejemplares adultos mantenidos en cautiverio, los que fueron capturados vivos en las costas de Iquique y Pisagua entre el 2001 y 2002. Los ejemplares, 7 hembras y 11 machos, se mantuvieron en estanque de $20.000 \mathrm{~L}$ de capacidad, con flujo continuo y conectado en la salida a un estanque colector de huevos. Los desoves ocurrieron de madrugada entre los meses de julio y noviembre 2005 a temperaturas entre los 14 a $17^{\circ} \mathrm{C}$. A 11,4 h post-fertilización y en etapa de gástrula los huevos fueron recolectados y puestos en incubación.

\section{Condiciones DE INCUBACIÓN}

Los huevos fueron dispuestos en grupos de 30 en frascos de vidrios de $40 \mathrm{ml}$ con agua de mar esterilizada por luz UV y filtrada a $0,2 \mu \mathrm{m}$ dentro de baños termoregulados a $12,14,16,18,20$ y $22^{\circ} \mathrm{C}\left( \pm 0,1^{\circ} \mathrm{C}\right)$, con 3 réplicas para cada tratamiento. Para los análisis de longitud de larvas, volumen de saco vitelino y 50\% de eclosión se utilizó el promedio de las réplicas, en el resto de los análisis se utilizaron todos los datos. Cada 1 h se registró el estadio de desarrollo y la mortalidad de los huevos. Una vez producida la eclosión de la totalidad de los huevos, las larvas fueron traspasadas con pipetas estériles a otros frascos con las mismas condiciones, donde se realizaron controles 2 veces al día para registrar el estado de desarrollo de las larvas, longitud corporal, consumo saco vitelo (volumen) y conteo de larvas muertas. Tanto en la fase de huevos como de larvas, los ejemplares colectados fueron fotografiados para luego realizar las mediciones a través del programa de procesamiento de imagen digital ImageJ (Rasband 1997-2008) ${ }^{1}$.

${ }^{1}$ Rasband W. 1997-2008. ImageJ 1.34s National Institutes of Health, Bethesda, USA. <http://rsb.info.nih.gov/ij/> 


\section{ANÁLISIS DE DATOS}

Para determinar el efecto de la temperatura sobre la tasa de desarrollo embrionario, se ajustó un modelo sigmoideo a la proporción de huevos eclosionados en función del tiempo para cada temperatura, de la forma:

$$
Y=\frac{a}{1+e^{b-c \cdot T}}
$$

donde, $a, b$ y $c$ son parámetros a estimar. $Y$ es la proporción de huevos eclosionados y $\mathrm{T}$ es el tiempo de incubación en horas.

También se consideró la relación entre el tiempo del $50 \%$ eclosión y la temperatura de incubación, a través de los modelos propuestos por Kamler (2002):

1) Modelo potencial: $Y=a T^{b}$

2) Modelo exponencial: $Y=a e^{b T}$

3) Modelo cuadrático: $Y=a+b T+c T^{2}$

4) Grados-días: $D^{\circ}=\Delta t^{*} T$

en donde, $Y$ es el tiempo del 50\% de eclosión en horas, $T$ es la temperatura de incubación $\left({ }^{\circ} \mathrm{C}\right), \Delta \mathrm{t}$ es el tiempo transcurrido (días) y $a, b$ y $c$ son constantes.

El éxito en la eclosión se determinó como el porcentaje del total de huevos eclosionados respecto de los huevos incubados al inicio, luego se ajustó una función polinomial de segundo orden al éxito de eclosión en función de la temperatura $\left({ }^{\circ} \mathrm{C}\right)$.

Para determinar el efecto de la temperatura sobre el consumo de vitelo, se calculó el volumen ( $V$ ) desde la fórmula para un esferoide, de acuerdo a:

$$
V=\frac{\pi}{6} L \times A^{2}
$$

donde $L$ es el largo (mm) y A el alto (mm) del saco de vitelo (Blaxter \& Hempel 1963, Hart \& Purser 1995, Baynes \& Howell 1996, Bermudes \& Ritar 1999, Watanabe et al. 1999).

Un modelo exponencial negativo se utilizó para el volumen del vitelo, en función de la temperatura y la supervivencia larval en función del tiempo, de la forma:

$$
Y=a \times e^{-b X}
$$

donde, $a$ y $b$ son parámetros y $X$ es la temperatura o tiempo según el caso.

\section{Resultados}

\section{HuEvos}

En todos los tratamientos, los parámetros estimados de la relación sigmoide entre la proporción de huevos eclosionados en función del tiempo de incubación, fueron significativamente distintos de $0(P<0,05$; Tabla 1),

\begin{abstract}
Tabla 1. Parámetros de la relación sigmoide entre la proporción de huevos eclosionados y el tiempo de incubación en Graus nigra. a, b y c: Parámetros; Sa, Sb y Sc: Error estándar de los parámetros; SCE: Suma cuadrado del error; gl: Grados de libertad; $\mathrm{t}$ : Prueba t de Student calculado; P: Probabilidad que el parámetro sea cero; $\mathbf{r}^{2}$ : Coeficiente de determinación / Parameters of the sigmoid relationship between the proportion of hatched eggs and incubation time in Graus nigra. $a, b$ and c: Parameters; Sa, Sb y Sc: Standard error of parameters; SCE: Sum of square errors; gl: Degrees of freedom; t: T-test calculator; $P$ : Probability of parameter equal zero; $r^{2}$ : Coefficient of determination
\end{abstract}

\begin{tabular}{ccccccc}
\hline Estadístico & $12^{\circ} \mathrm{C}$ & $14^{\circ} \mathrm{C}$ & $16^{\circ} \mathrm{C}$ & $18^{\circ} \mathrm{C}$ & $20^{\circ} \mathrm{C}$ & $22^{\circ} \mathrm{C}$ \\
\hline $\mathrm{a}$ & 0,570 & 0,985 & 0,888 & 0,883 & 0,681 & 0,329 \\
$\mathrm{Sa}$ & 0,0278 & 0,0224 & 0,0184 & 0,0458 & 0,0426 & 0,0088 \\
$\mathrm{~b}$ & 14,579 & 44,719 & 47,240 & 19,017 & 20,853 & 16,976 \\
$\mathrm{Sb}$ & 6,3717 & 0,6849 & 0,8372 & 4,6543 & 5,7182 & 6,9758 \\
$\mathrm{c}$ & 0,223 & 0,872 & 1,330 & 0,596 & 0,738 & 0,728 \\
$\mathrm{Sc}$ & 0,0982 & 0,0126 & 0,0254 & 0,1439 & 0,2127 & 0,3006 \\
$\mathrm{SCE}$ & 1,5037 & 0,3561 & 0,1162 & 1,3842 & 1,2097 & 0,0693 \\
$\mathrm{gl}$ & 209 & 76 & 30 & 123 & 87 & 78 \\
$\mathrm{r}{ }^{2}$ & 0,75 & 0,97 & 0,98 & 0,88 & 0,82 & 0,95 \\
$\mathrm{t}: \mathrm{Ho}: \mathrm{a}=0$ & 20,52 & 43,99 & 48,21 & 19,29 & 15,97 & 37,33 \\
$P($ Ho: $\mathrm{a}=0)$ & $5,55 \mathrm{E}-52$ & $1,25 \mathrm{E}-107$ & $3,37 \mathrm{E}-115$ & $2,72 \mathrm{E}-48$ & $4,64 \mathrm{E}-38$ & $2,11 \mathrm{E}-94$ \\
$\mathrm{t}:$ Ho: $\mathrm{c}=0$ & 2,27 & 69,04 & 52,41 & 4,14 & 3,68 & 2,42 \\
$P($ Ho: $\mathrm{c}=0)$ & $2,44 \mathrm{E}-02$ & $7,59 \mathrm{E}-146$ & $3,32 \mathrm{E}-122$ & $4,97 \mathrm{E}-05$ & $2,95 \mathrm{E}-04$ & $1,63 \mathrm{E}-02$ \\
\hline
\end{tabular}



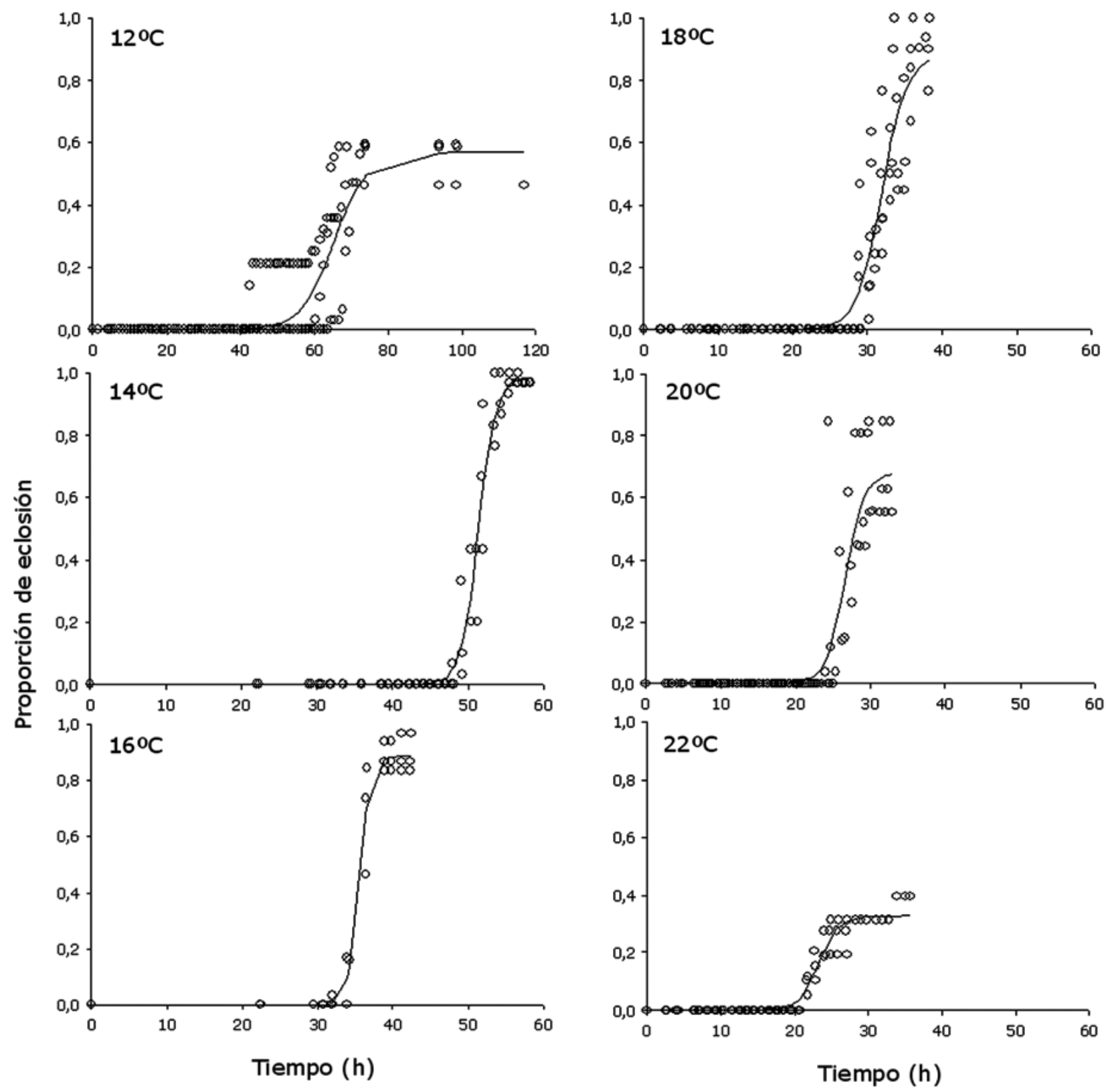

Figura 1. Proporción de huevos eclosionados de Graus nigra en función del tiempo $(\mathrm{h})$ bajo distintas temperaturas $\left({ }^{\circ} \mathrm{C}\right)$. Nótese la diferencia de escala en eje $\mathrm{X}$ sólo en figura de $12^{\circ} \mathrm{C}$ para una mejor representación / Proportion of the hatched eggs of Graus nigra in function of time (h) under different temperatures $\left({ }^{\circ} \mathrm{C}\right)$. Scale of $\mathrm{X}$ axis changed only in figure of $12^{\circ} \mathrm{C}$ for a better representation

existiendo un claro acortamiento del tiempo a la eclosión total a medida que aumentó la temperatura (Fig. 1). Los porcentajes de huevos eclosionados (éxito en la eclosión) en función de la temperatura muestra una relación parabólica que explica el 87\% de la varianza (Fig. 2; Tabla 2), con un máximo estimado en torno a los $16^{\circ} \mathrm{C}$.
El desarrollo embrionario se aceleró con el incremento de la temperatura, especialmente entre los 12 y $16^{\circ} \mathrm{C}$ que muestran una mayor pendiente (Fig. 3). Si bien, todos los ajustes explican sobre el 90\% de la varianza de la tasa de desarrollo embrionario y sus parámetros difieren significativamente de cero $(P<0,05$; Tabla 3$)$, el modelo 


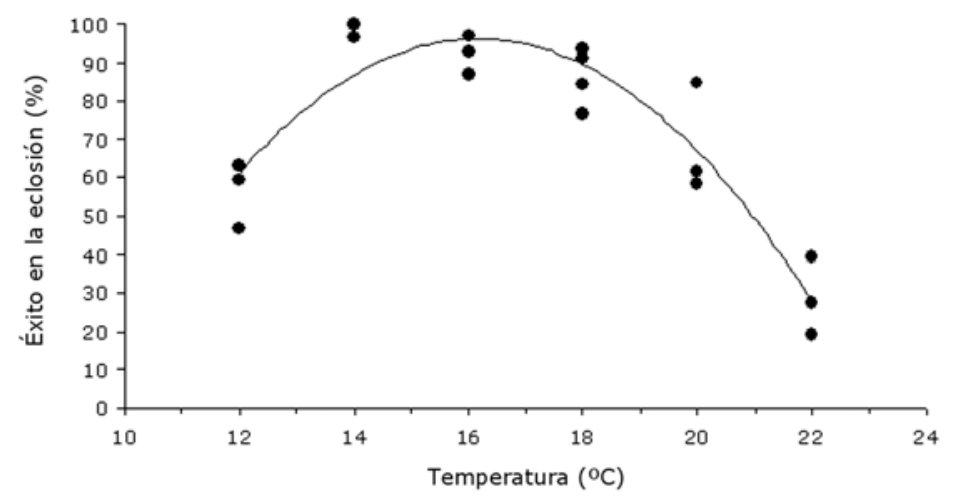

Figura 2. Porcentaje de eclosión para huevos incubados a diferentes temperaturas. Línea es ajuste de función parabólica / Hatched eggs percentage at incubated at different temperatures. Line is a parabolic fitted function

Tabla 2. Parámetros del ajuste de la relación parabólica entre la temperatura y el éxito de eclosión en Graus nigra. a, b y c: Parámetros; SCE: Suma cuadrado del error; $n$ : Números de huevos; t: Prueba t de Student calculado; $P$ : Probabilidad que el parámetro sea cero; $\mathbf{r}^{2}$ : Coeficiente de determinación / Parameters of the parabolic relationship between hatching success and temperature in Graus nigra. a, b y c: Parameters; SCE: Sum of the squared errors; $n$ : Egg numbers; $t$ : T-test calculator; $P$ : Probability of parameter equal zero; $r^{2}$ : Coefficient of determination

\begin{tabular}{cccc}
\hline Resumen & $\mathrm{a}$ & $\mathrm{b}$ & $\mathrm{c}$ \\
\hline Estimado & $-429,75$ & 65,04 & $-2,01$ \\
Error estándar & 63,32 & 7,65 & 0,22 \\
$\mathrm{t}$ & $-6,79$ & $-8,95$ & 8,50 \\
$P$ & 0 & 0 & 0 \\
$\mathrm{SCE}$ & 1532,73 & & \\
$\mathrm{r}^{2}$ & 0,87 & & \\
$\mathrm{n}$ & 19 & & \\
\hline
\end{tabular}

cuadrático presenta un mejor ajuste (menor suma residual). En términos de grados-día se observa que entre los 16 y $22^{\circ} \mathrm{C}$ se mantiene relativamente estable.

\section{LARVAS}

El consumo del vitelo de las larvas se aceleró con el incremento de la temperatura (Fig. 4, Tabla 4), terminándose a las $129 \mathrm{~h}$ para los $12^{\circ} \mathrm{C}$ y a las $49 \mathrm{~h}$ para los 18 y $20^{\circ} \mathrm{C}$. No se pudo establecer el término en el consumo de vitelo para 16 y $22^{\circ} \mathrm{C}$. Sin embargo, se produce una retracción en la longitud larval antes que se consuma totalmente el vitelo a temperaturas superiores a los $18^{\circ} \mathrm{C}$ (Fig. 5).

La disminución en el tiempo del número de larvas, debido principalmente a la inanición producto del agotamiento del vitelo, se ajusta a un modelo exponencial (Tabla 5, Fig. 6), excepto para los $22^{\circ} \mathrm{C}$ debido a su corta duración. Se observan además diferencias en la tendencia entre las réplicas, especialmente a $18^{\circ} \mathrm{C}$, producto posiblemente de condiciones de cultivo y de huevos.

\section{Discusión}

El manejo y conservación de cualquier población de peces requiere de un entendimiento de todo el ciclo de vida, incluyendo huevos y larvas (Strydom 2008). Esto es fundamental ya que la gran mayoría de peces costeros del litoral de Chile se encuentran desprotegidos de cualquier medida de regulación para su conservación o manejo. Según Godoy et al. (2010), la pesca submarina no reglamentada ha agotado los peces en los ecosistemas 


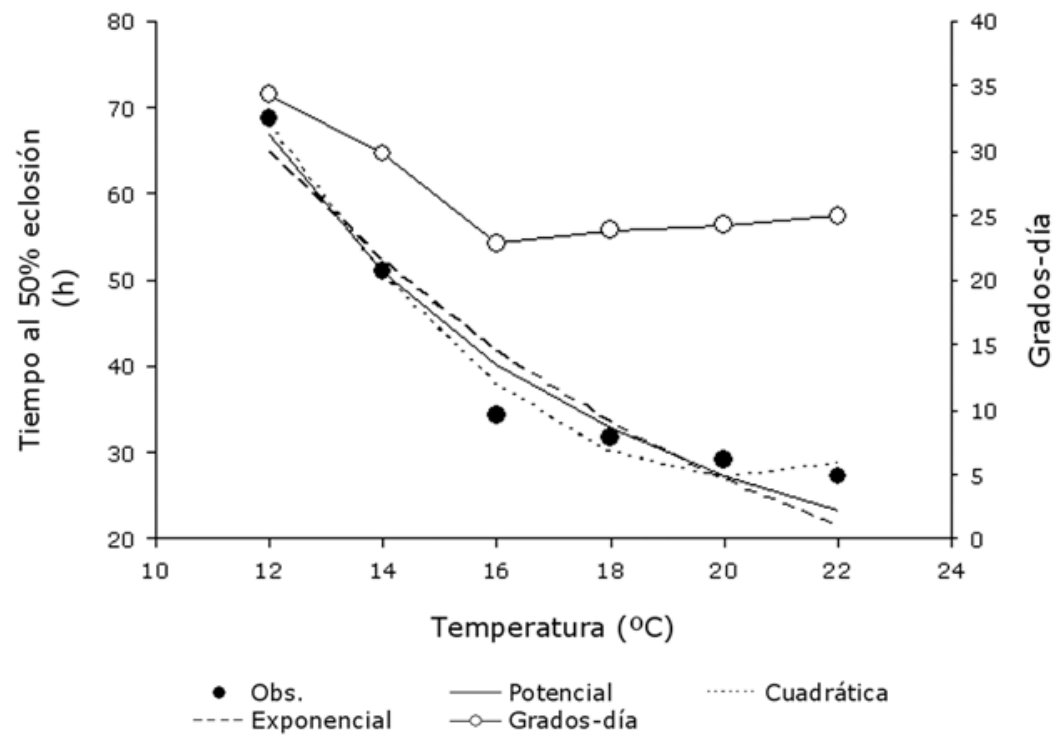

Figura 3. Relación entre la temperatura y el tiempo al $50 \%$ de eclosión en huevos de Graus nigra. Líneas corresponden al ajuste de 3 modelos (potencial, cuadrático y exponencial) y línea con círculos blancos grados-días / Relationship between time to $50 \%$ hatch and temperature for Graus nigra eggs. Lines correspond to 3 fitted model (power, quadratic, and exponential) and line with white circles degree-days

Tabla 3. Parámetros de la relación entre el tiempo al $50 \%$ de eclosión y el tiempo de incubación en Graus nigra. a, b y c: Parámetros; SCE: Suma cuadrado del error; gl: Grados de libertad; t: Prueba t de Student calculado; P: Probabilidad que el parámetro sea igual a cero; $\mathbf{r}^{2}$ : Coeficiente de determinación / Parameters of the relationship between the time to $50 \%$ hatch and the incubation time in Graus nigra. a, b y c: Parameters; SCE: Sum of the squared errors; gl: Degrees of freedom; $t$ : T-test calculator; $P$ : Probability of parameter equal cero; $r^{2}$ : Coefficient of determination

\begin{tabular}{cccccccc}
\hline Modelo & Parámetros & Estimado & Error estándar & $\mathrm{t}$ & $\mathrm{p}$ & $\mathrm{SCE}$ & $\mathrm{r}^{2}$ \\
\hline \multirow{2}{*}{ Potencial } & $\mathrm{a}$ & 51712,73 & 2722,16 & 1,900 & 0,130 & 61,66 & 0,95 \\
& $\mathrm{~b}$ & $-1,75$ & 0,20 & $-8,891$ & 0,001 & & \\
Exponencial & $\mathrm{a}$ & 243,89 & 63,35 & 3,850 & 0,018 & 110,98 & 0,92 \\
& $\mathrm{~b}$ & $-0,11$ & 0,02 & $-6,358$ & 0,003 & & \\
Cuadrática & $\mathrm{a}$ & 274,02 & 32,58 & 8,411 & 0,004 & 23,933 & 0,98 \\
& $\mathrm{~b}$ & $-24,38$ & 3,94 & $-6,183$ & 0,009 & & \\
& $\mathrm{c}$ & 0,60 & 0,12 & 5,204 & 0,014 & & \\
\hline
\end{tabular}

templados costeros de Chile y también ha provocado cambios en la composición de las capturas en el tiempo, donde las 3 especies de mayor tamaño, como son Graus nigra (mulata), Semicossyphus darwini (pejeperro) y Medialuna ancietae (acha), han disminuido drásticamente a partir de finales de la década de 1990.
La temperatura óptima estimada para el éxito en la eclosión y supervivencia larval fue de $16^{\circ} \mathrm{C}$, lo que coincide con la temperatura de mayor intensidad de desove de los reproductores mantenidos en cautiverio (Futagawa 2006²). Según Kamler (2002), los adultos seleccionarían el ambiente donde desovan, lo que también implicaría una

${ }^{2}$ Futagawa M. 2006. Optimum water temperature for maturation of Graus nigra. XXVI Congreso de Ciencias del Mar, IquiqueChile, 22-26 de mayo, p. 154. 


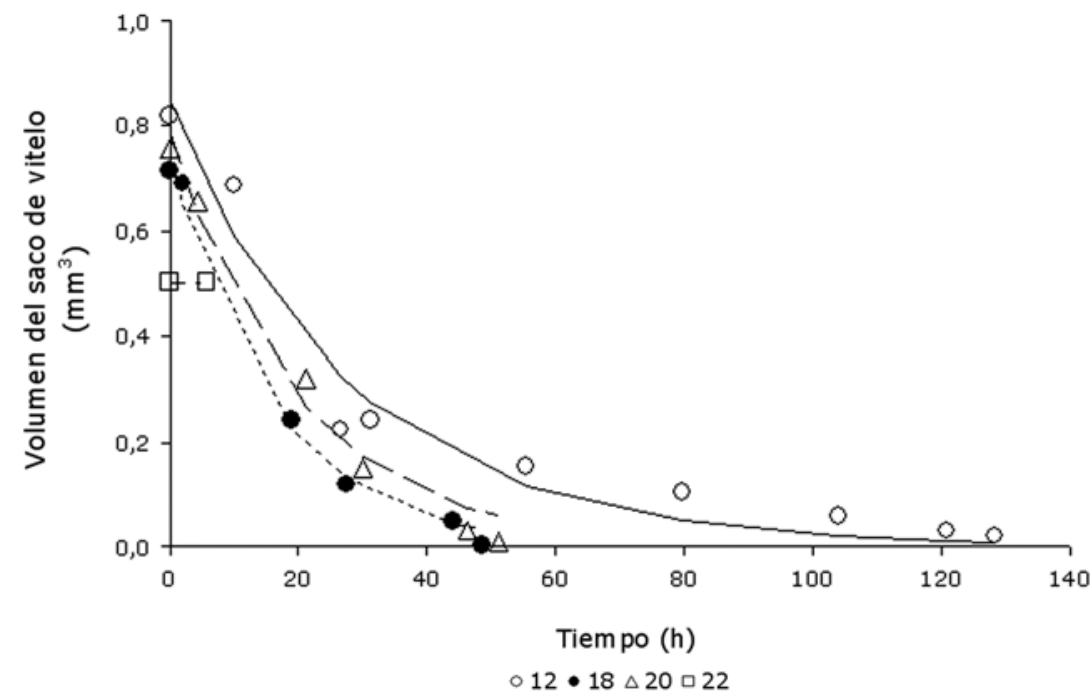

Figura 4. Volumen del saco vitelino en función del tiempo bajo diferentes temperaturas $\left(12,18,20\right.$ y $\left.22^{\circ} \mathrm{C}\right) /$ Yolk-sac volume in function of time under different temperatures $\left(12,18,20\right.$ and $\left.22^{\circ} \mathrm{C}\right)$

Tabla 4. Parámetros de la relación exponencial negativa entre el volumen del vitelo y el tiempo de incubación a distintas temperatura en Graus nigra. a y b: Parámetros; Sa y Sb: Error estándar de los parámetros; SCE: Suma cuadrado del error; gl: Grados de libertad; $t$ : Prueba $t$ de Student calculado; $P$ : Probabilidad que el parámetro sea igual a cero; $r^{2}$ : Coeficiente de determinación / Parameters of the exponential negative relationship between yolk-sac volume and the incubation time for different temperature in Graus nigra. a y b: Parameters; Sa y Sb: Standard error of parameter; SCE: Sum of the squared errors; gl: Degrees of freedom; t: T-test calculator; $P$ : Probability of parameter equal zero; $r^{2}$ : Coefficient of determination

\begin{tabular}{cccc}
\hline Estadístico & $12^{\circ} \mathrm{C}$ & $18^{\circ} \mathrm{C}$ & $20^{\circ} \mathrm{C}$ \\
\hline $\mathrm{a}$ & 0,849 & 0,750 & 0,790 \\
$\mathrm{Sa}$ & 0,0598 & 0,0235 & 0,0403 \\
$\mathrm{~b}$ & 0,036 & 0,063 & 0,051 \\
$\mathrm{Sb}$ & 0,0051 & 0,0051 & 0,0059 \\
$\mathrm{SCE}$ & 0,0307 & 0,0036 & 0,0092 \\
$\mathrm{gl}$ & 7 & 4 & 4 \\
$\mathrm{t}: \mathrm{Ho}: \mathrm{b}=0$ & 7,10 & 12,35 & 8,69 \\
$P$ & $1,94 \mathrm{E}-04$ & $2,47 \mathrm{E}-04$ & $9,64 \mathrm{E}-04$ \\
$\mathrm{r}^{2}$ & 0,96 & 0,99 & 0,98 \\
\hline
\end{tabular}

temperatura adecuada para la incubación de huevos y larvas, ya que sería un factor controlador de la tasa de desarrollo y supervivencia, favoreciéndolas dentro de cierto rango óptimo, pero actuando como agente letal fuera de éste (Pepin 1991, Hart \& Purser 1995, Kamler $1992,1998,2002)$. Las temperaturas $<16^{\circ} \mathrm{C}$, inducen tasas de desarrollo embrionario y larval más lentas, lo que en el ambiente representaría una mayor vulnerabilidad a la depredación. En cambio temperaturas $>16^{\circ} \mathrm{C}$, si bien incrementaron las tasas de desarrollo, se observaron eclosiones prematuras de embriones más pequeños y menos desarrollados posiblemente por la secreción temprana de enzimas (Hayes et al. 1953) en la eclosión y el incremento en la movilidad del embrión (Kamler 1998, Jordaan \& Kling 2003). La disminución en el tamaño de las larvas estaría asociada a la inanición producto de la 

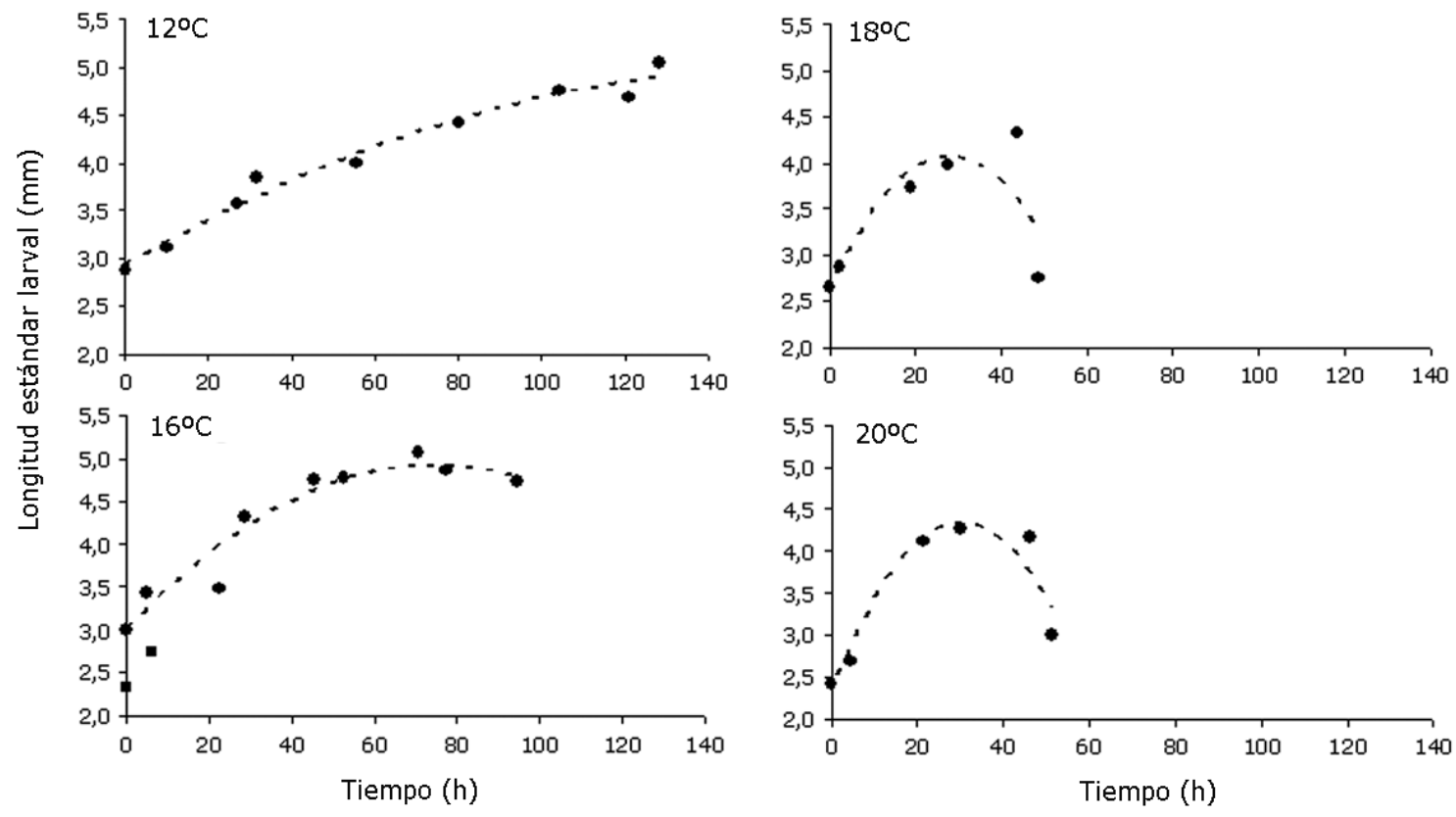

Figura 5. Longitud larval $(\mathrm{mm})$ en función del tiempo para diferentes temperaturas $\left(12,16,18\right.$ y $\left.20^{\circ} \mathrm{C}\right) /$ Larval length $(\mathrm{mm})$ in function of time for different temperatures $\left(12,16,18\right.$ and $\left.20^{\circ} \mathrm{C}\right)$

Tabla 5. Parámetros de la relación entre el número de larvas y su edad para distintas temperaturas en Graus nigra. a y b: Parámetros; Sa y Sb: Error estándar de los parámetros; SCE: Suma cuadrado del error; gl: Grados de libertad; t: Prueba t de Student calculado; P: Probabilidad que el parámetro sea igual a cero; $r^{2}$ : Coeficiente de determinación. El ajuste para $22^{\circ} \mathrm{C}$ no es significativo / Parameters of the relationship between the numbers of larvae and its age for different temperature in Graus nigra. a and b: Parameters; Sa y Sb: Standard error of parameters; SCE: Sum of square errors; gl: Degrees of freedom; t: T-test calculator, $P$ : Probability for parameter equal zero; $r^{2}$ : Coefficient of determination. $22^{\circ} \mathrm{C}$ is not significant

\begin{tabular}{ccccccc}
\hline Estadistico & $12^{\circ} \mathrm{C}$ & $14^{\circ} \mathrm{C}$ & $16^{\circ} \mathrm{C}$ & $18^{\circ} \mathrm{C}$ & $20^{\circ} \mathrm{C}$ & $22^{\circ} \mathrm{C}$ \\
\hline $\mathrm{a}$ & 16,864 & 30,914 & 28,184 & 21,703 & 18,655 & 8,536 \\
$\mathrm{Sa}$ & 0,8980 & 1,8969 & 2,3036 & 1,5638 & 1,1472 & 1,8153 \\
$\mathrm{~b}$ & 0,005 & 0,007 & 0,019 & 0,012 & 0,032 & 0,019 \\
$\mathrm{Sb}$ & 0,0005 & 0,0010 & 0,0041 & 0,0026 & 0,0047 & 0,0257 \\
$\mathrm{SCE}$ & 189,43900 & 541,59000 & 713,00300 & 2229,41000 & 152,90900 & 232,74700 \\
$\mathrm{gl}$ & 33 & 25 & 20 & 58 & 20 & 10 \\
$\mathrm{t}: \mathrm{Ho}: \mathrm{b}=0$ & 8,74 & 6,89 & 4,55 & 4,81 & 6,88 & 0,75 \\
$P$ & $4,25 \mathrm{E}-10$ & $3,22 \mathrm{E}-07$ & $1,93 \mathrm{E}-04$ & $1,11 \mathrm{E}-05$ & $1,11 \mathrm{E}-06$ & 0,47 \\
$\mathrm{r}^{2}$ & 0,80 & 0,73 & 0,66 & 0,45 & 0,85 & $7 \mathrm{E}-02$ \\
\hline
\end{tabular}



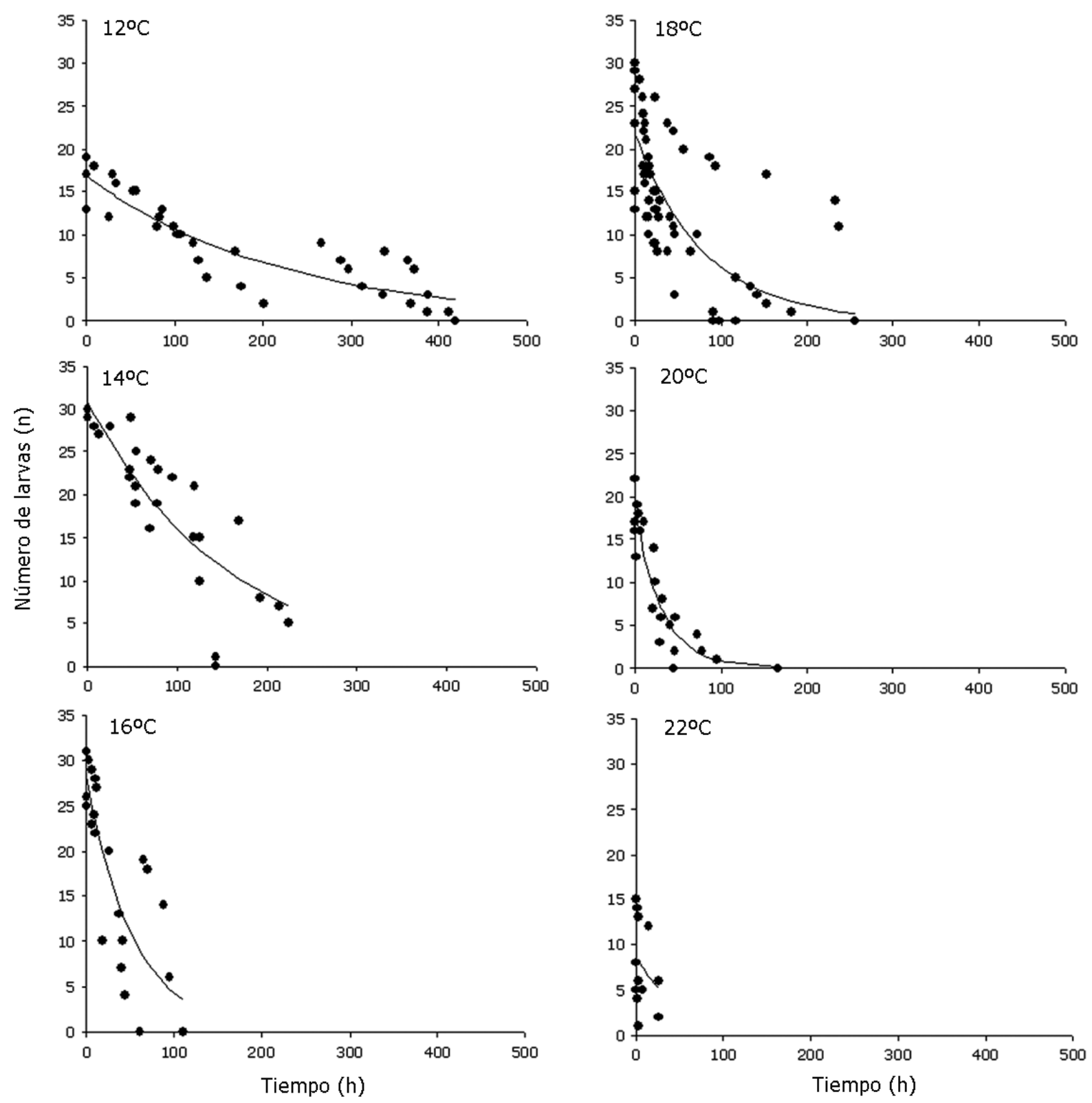

Figura 6. Supervivencia larval temprana a distintas temperaturas en Graus nigra / Early larval survival at different temperature in Graus nigra 
absorción del vitelo y el no encontrar alimento en la condiciones de este estudio, por lo que la larva sufre un déficit de energía y al no alimentarse, comienza la autolisis (Lasker 1965, Theilacker \& Dorsey 1980).

Los cambios de temperatura en la naturaleza no suelen ser suficiente para causar la mortalidad por trastornos del desarrollo (mortalidad directa), ya que las anomalías de temperatura superficial del mar por lo general son del orden de $4^{\circ} \mathrm{C}$ (Pepin 1991). Sin embargo, eventos de baja frecuencia como evento El Niño que en la costa de la zona norte de Chile se manifiestan con anomalías térmicas de hasta $5,5^{\circ} \mathrm{C}$ (Fuenzalida et al. 1999) podrían tener un impacto negativo en el desarrollo de estadios tempranos de esta especie. No obstante, las repuestas ecofisiológicas a eventos oceanográficos como El Niño, han sido poco estudiadas debido principalmente a la carencia de series de tiempo de variables biológicas que permita contrastar su comportamiento antes, durante y después de ocurrido el evento. No obstante, son múltiples las posibles respuestas que la fauna presentaría frente a las variaciones en las condiciones ambientales, entre las que se cuentan cambios en las densidades poblacionales, mortalidad, migraciones, parámetros reproductivos, en los rangos de distribución latitudinal, longitudinal y en profundidad (Tarazona et al. 1985, Soto 1985, Berrios \& Vargas 2000, Hernández et al. 2002, Sielfeld et al. 2002, Mendo \& Wolf 2003, Gárate \& Pacheco 2004), entre otras.

Los resultados obtenidos en el presente estudio entregan información básica para su aplicación en el cultivo con fines comerciales o de repoblamiento, en una especie que muestra importantes signos de agotamiento de su población (Godoy et al. 2010). Sin embargo, queda aún por determinar los efectos combinados con otros factores extrínsecos como el oxígeno, salinidad, pH, etc. y factores intrínsecos como condición de los reproductores, tamaño y calidad de los huevos.

\section{Agradecimientos}

Muchas de las horas sin dormir que implicó el trabajo experimental fueron cubiertas por Fernando Yañez B. (Q.E.P.D), quien falleció buceando en septiembre del año 2007. Todos los que tuvimos la suerte de conocerlo nunca lo olvidaremos. Este modesto trabajo está dedicado a su memoria. El presente estudio fue realizado al amparo del Magíster en Ciencias Aplicadas mención Biología Pesquera de la Universidad Arturo Prat.

\section{LITERATURA CITADA}

Arul V. 1991. Effects of temperature on yolk utilization of Channa striatus. Journal of Thermal Biology 16: 1-5.

Bagarinao T. 1986. Yolk resorption, onset of feeding and survival potential of larvae of three tropical marine fish species reared in the hatchery. Marine Biology 91: 449-459.

Baynes SM \& BR Howell. 1996. The influence of egg size and incubation temperature on the condition of the Solea solea (L.) larvae at hatching and first feeding. Journal of Experimental Marine Biology and Ecology 199: 59-77.

Beacham TD \& CB Murray. 1990. Temperature egg size, and development of embryos and alevins of five species of pacific salmon: a comparative analysis. Transactions of the American Fisheries Society 119: 927-945.

Bermudes M \& AJ Ritar. 1999. Effects of temperature on the embryonic development of the striped trumpeter (Latris lineate Bloch and Schneider, 1801). Aquaculture 176: 245-255.

Berrios V \& M Vargas. 2000. Estructura del ensamble de peces intermareales de la costa rocosa del norte de Chile. Revista de Biología Marina y Oceanografía 35: 73-81.

Blaxter JHS. 1992. The effect of temperature on larval fishes. Netherlands Journal of Zoology 42: 336-357.

Blaxter JHS \& G Hempel. 1963. The influence of egg size on herring larvae (Clupea harengus L). Journal du Conseil International pour l' Exploration de la Mer 28: 211-240.

Bolla S \& I Holmefjord. 1988. Effect of temperature and light on development of Atlantic halibut larvae. Aquaculture 74: 355-358.

Buckley LJ, TM Bradley \& J Allen-Guilmette. 2000. Production, quality, and low temperature incubation of eggs of Atlantic cod Gadus morhua and haddock Melanogrammus aeglefinus in captivity. Journal of the World Aquaculture Society 31: 22-29.

Camus P \& C Koutsikopoulos. 1984. Incubation experimentale et developpement embryonnaire de la daurade royale, Sparus aurata, a differant temperatures. Aquaculture 42: 117-128.

Cushing DH. 1973. The dependence of recruitment on parent stock. Journal of the Fisheries Research Board of Canada 30: 1965-1976.

Flores H \& J Rendic. 2011. Conducta alimentaria, supervivencia y crecimiento de juveniles silvestres de Graus nigra Philippi, 1887 en cautiverio (Perciformes: Kyphosidae). Latin American Journal of Aquatic Research 39(3): 607-612.

Flores H \& A Smith. 2010. Biología reproductiva de Graus nigra (Perciformes, Kyphosidae) en las costas del norte de Chile. Revista de Biología Marina y Oceanografía 45(1): 659-670.

Fuenzalida R, J Blanco \& S Hormazabal. 1999. Efectos oceanográficos y metereológicos durante el evento el niño 1997-98, en la zona de Iquique $\left(20^{\circ} \mathrm{S}\right)$. Investigaciones Científicas y Tecnológicas, Serie Ciencias del Mar 4: 1-3. 
Galloway TF, E Kjørsvik \& H Kryvi. 1998. Effect of temperature on viability and axial muscle development in embryos and yolk sac larvae of the Northeast Artic cod (Gadus morhua). Marine Biology 132: 559-567.

Garate A \& A Pacheco. 2004. Distribution changes and abundance of shallow water ictiofauna in San Bartolo (Lima, Perú) after the ENSO 1997-1998. Ecología Aplicada, Perú 3: $148-153$.

Godoy N, S Gelcich, JA Vasquez \& J Castilla. 2010. Spearfishing to depletion: evidence from temperate reef fishes in Chile. Ecological Applications 20(6): 1504-1511.

Hardy RS \& MK Litvak. 2004. Effects of temperature on the early development, growth and survival of shortnose sturgeon, Acipenser brevirostrum, and Atlantic sturgeon, Acipenser oxyrhynchus, yolk-sac larvae. Environmental Biology of Fishes 70: 145-154.

Hart PR \& GJ Purser. 1995. Effects of salinity and temperature on eggs and yolk sac larvae of the greenback flounder (Rhombosolea tapirina Günther, 1982). Aquaculture 136: 221-230.

Hayes F, D Pelluet \& E Gorham. 1953. Some effects of temperature on the embryonic development of the salmon (Salmo salar). Canadian Journal of Zoology 31: 42-51.

Hernandez CE, PE Neill, JM Pulgar, FP Ojeda \& F Bozinovic. 2002. Water temperature fluctuations and territoriality in the intertidal zone: two possible explanations for the elevational distribution of body size in Graus nigra. Journal of Fish Biology 61: 472-488.

Hjort J. 1914. Fluctuations in the great fisheries of northern Europe viewed in the light of biological research. Rapports et Proces-Verbaux Des Reunions, Conseil International pour l'Exploration de la Mer 20: 1-228.

Howell WH. 1980. Temperature effects on growth and yolk utilization in yellowtail flounder, Limanda ferruginea, yolksac larvae. Fishery Bulletin 78: 731-739.

Jensen AJ, BO Johnsen \& L Saksgård. 1989. Temperature requirements in Atlantic salmon (Salmo salar), brown trout (Salmo trutta), and Arctic char (Salvelinus alpinus) from hatching to initial feeding compared with geographic distribution. Canadian Journal of Fisheries and Aquatic Sciences 46: 786-789.

Jordaan A \& LJ Kling. 2003. Determining the optimal temperature range for Atlantic cod (Gadus morhua) during early life. In: Browman HI \& AB Skiftesvik (eds). The Big Fish Bang. Proceedings of the 26th Annual Larval Fish Conference, Institute of Marine Research, Bergen, pp. 45-62.

Kamler E. 1992. Early life history of fish: An energetics approach, 267 pp. Chapman \& Hall, London.
Kamler E. 2002. Ontogeny of yolk-feeding fish: an ecological perspective. Reviews in Fish Biology and Fisheries 12(1): 79-103.

Kamler E, H Keckeis \& E Bauer-Nemeschkal. 1998. Temperature- induced changes of survival, development and yolk partitioning in Chondrostoma nasus. Journal of Fish Biology 53: 658-682.

Klimogianni A, G Koumoundouros, P Kaspiris \& M Kentouri. 2004. Effect of temperature on the egg and yolksac larval development of common pandora, Pagellus erythrinus. Marine Biology 145: 1015-1022.

Lasker R. 1965. The physiology of Pacific sardine embryos and larvae. California Cooperative Oceanic Fisheries Investigations and Reports 10: 96-101.

Mendo J \& M Wolff. 2003. El impacto de El Niño sobre la producción de concha de abanico (Argopecten purpuratus) en Bahía Independencia, Pisco, Perú. Ecología Aplicada 2(1): 51-57.

Muñoz AA \& FP Ojeda. 1997. Feeding guild structure of a rocky intertidal ûsh assemblage in central Chile. Environmental Biology of Fishes 49: 471-479.

Muñoz AA \& FP Ojeda. 1998. Guild structure of carnivorous intertidal shes of the Chilean coast: implications of ontogenetic dietary shifts. Oecologia 114: 563-573.

Muñoz A, E Segovia \& H Flores. 2012. Deshabituación alimentaría y crecimiento de juveniles de Graus nigra (Philippi, 1887) en condiciones de cultivo. Latin American Journal of Aquatic Research 40(3): 578-583.

Ojanguren AF, FG Reyes-Gavilan \& R Rodríguez-Muñoz. 1999. Effects of temperature on growth and efficiency of yolk utilisation in eggs and pre-feeding larval stages of Atlantic salmon. Aquaculture International 7: 81-87.

Parra G \& M Yúfera. 2001. Comparative energetics during early development of two marine fish species, Solea senegalensis (Kaup) and Sparus aurata (L.). Journal of Experimental Biology 204: 2175-2183.

Pepin P. 1991. Effect of temperature and size on development, mortality, and survival rates of the pelagic early life history stages of marine fish. Canadian Journal of Fisheries and Aquatic Sciences 48: 503-518.

Polo A, M Yufera \& E Pascual. 1991. Effects of temperature on egg and larval development on Sparus aurata L., Aquaculture 92: 367-375.

Sielfeld W, M Vargas, V Berrios \& G Aguirre. 2002. Warm ENSO events and their effects on the coastal fish fauna of northern Chile. Investigaciones Marinas 30(1): 122-124.

Soto R. 1985. Efectos del fenómeno el Niño 1982-83 en los ecosistemas de la I región. Investigación Pesquera 32: 199206. 
Stepien CA. 1990. Population structure, diets and biogeographic relationship of a rocky intertidal sh assemblage in central Chile: high levels of herbivory in a temperate system. Bulletin of Marine Science 47: 598-612.

Strydom NA. 2008. Utilization of shallow subtidal bays associated with warm temperate rocky shores by the latestage larvae of some inshore fish species, South Africa. African Zoology 43: 205-269.

Tarazona J, C Paredes, L Romero, V Blaskovich, S Guzman \& S Sanchez. 1985. Características de la vida planctónica y colonización de los organismos epilíticos durante el fenómenos 'El Niño'. En: Arntz W, A Landa \& J Tarazona (eds). El Niño y su impacto en la fauna marina. Boletín del Instituto del Mar del Perú-Callao, Vol. extraordinario: 41-50.
Theilacker GH \& K Dorsey. 1980. Larval fish diversity, a summary of laboratory and field research. In: Workshop on the effects of environmental variation on the survival of larval pelagic fishes. IOC-UNESCO, Workshop Report 28: 105-142.

Varas E \& FP Ojeda. 1990. Intertidal sh assemblage of the central Chilean coast: diversity, abundance and trophic patterns. Revista de Biología Marina 25: 59-70.

Watanabe C, T Hanai, K Meguro, R Ogino, Y Kubota \& R Kimura. 1999. Spawning biomass estimates of chub mackerel Scomber japonicus of Pacific subpopulation off central Japan by a daily egg production method. Nippon Suisan Gakkaishi 65(4):695-702.

Recibido el 8 de agosto de 2013 y aceptado el 12 de marzo de 2014

Editor Asociado: Mauricio Landaeta D. 